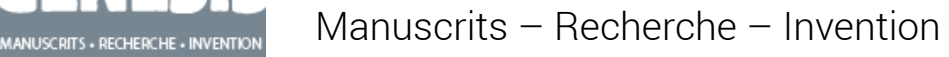

$53 \mid 2021$

Sartre Beauvoir. Genèses croisées

\title{
Genèse du « Spleen de Paris »
}

\section{Aurélia Cervoni}

\section{OpenEdition}

\section{Journals}

Édition électronique

URL : https://journals.openedition.org/genesis/6307

DOI : 10.4000/genesis.6307

ISSN : 2268-1590

\section{Éditeur :}

Presses universitaires de Paris Sorbonne (PUPS), Société internationale de génétique artistique littéraire et scientifique (SIGALES)

\section{Édition imprimée}

Date de publication : 15 décembre 2021

Pagination : 201-206

ISBN : 979-10-231-0716-6

ISSN : 1167-5101

\section{Référence électronique}

Aurélia Cervoni, «Genèse du « Spleen de Paris » », Genesis [En ligne], 53| 2021, mis en ligne le 15 décembre 2021, consulté le 13 janvier 2023. URL : http://journals.openedition.org/genesis/6307 ; DOI : https://doi.org/10.4000/genesis.6307 


\title{
Genèse du "Spleen de Paris"
}

\author{
Aurélia Cervoni
}

É diter Le Spleen de Paris soulève de nombreuses difficultés. Baudelaire est mort sans avoir eu le temps d'achever son recueil de poèmes en prose. Les notes de travail retrouvées dans ses papiers donnent un aperçu des sujets qu'il envisageait de traiter. On y relève une centaine de titres aux résonances suggestives : Aux philosophes amateurs de bals masqués, Le Séduisant Croque-mort, La Poule noire, La Cour des messageries, Les Reproches du portrait, La Fin du monde, Les Aliénistes, Le Poisson rouge, La Salle des martyrs, L'Homme aux diamants, Le Vieil Entreteneur, Avant d'être mûr, Les Deux Ivrognes, L'Orgue de Barbarie, L'Autel de Moloch...1.

«Fiction critique», selon la formule de Jacques Dupont ${ }^{2}$, Le Spleen de Paris est en premier lieu une construction due aux premiers éditeurs de Baudelaire, Banville et Asselineau, qui ont supervisé l'édition posthume des Euvres complètes du poète chez Michel Lévy en 1868-1870.

\section{L'architecture du recueil}

La plupart des cinquante poèmes qui composent Le Spleen de Paris tel que nous le connaissons ont paru dans des recueils collectifs et dans la presse entre 1855 et 1867 . Pour en établir l'ordre, Banville et Asselineau se sont fondés sur une liste de titres manuscrite actuellement conservée à la Bibliothèque littéraire Jacques-Doucet sous la cote Alpha 9022 et dont nous donnons ici la transcription ${ }^{3}$ :

1. L'Étranger

2. Le Désespoir de la vieille

3. Le Confiteor de l'artiste

4. Un plaisant

5. La Chambre double

6. Chacun sa Chimère

7. Le Fou et la Vénus

8. Le Chien et le Flacon

9. Le Mauvais Vitrier

10. À une heure du matin

11. La Femme sauvage et la petite-maîtresse

12. Les Foules

13. Les Veuves
14. Le Vieux Saltimbanque

15. Le Gâteau

16. L'Horloge

17. Un hémisphère dans une chevelure

18. L'Invitation au voyage

19. Le Joujou du pauvre

20. Les Dons des Fées

21. Éros, Plutus et la Gloire

22. Le Crépuscule du soir

23. La Solitude

24. Les Projets

25. La Belle Dorothée

26. Les Yeux des pauvres

27. Une mort héroïque

28. La Fausse Monnaie

29. Le Joueur généreux

30. La Corde

31. Les Vocations

32. Le Thyrse

33. Enivrez-vous

34. Déjà

35. Les Fenêtres

36. Le Désir de peindre

37. Les Bienfaits de la lune (boulevard)

38. Laquelle est la vraie? (boulevard)

39. Un cheval de race

40. Le Miroir +

41. Le Port +

42. Portraits de maîtresses (inédit)

43. Le Galant Tireur (inédit)

44. La Soupe et les nuages (inédit)

45. Le Tir et le cimetière (inédit)

46. Perte d'auréole (inédit)

47. Mademoiselle Bistouri (inédit)

48. Anywhere, out of the world (inédit)

49. Assommons les pauvres (inédit)

50. Les Bons Chiens (inédit)

Chaque pièce en pages

1. Voir ces listes de titres dans $C E C I$, éd. cit., p. 366-370.

2. Jacques Dupont, «Le Spleen de Paris, une fiction critique?», L'Année Baudelaire, no 16, 2012, p. 41-53. 
Cette liste est-elle réellement une table des matières ? On observe que les mots «table» ou «sommaire» n'y figurent pas. Récemment, Jacques Dupont a émis l'hypothèse que ce document serait un «aide-mémoire ${ }^{4} »$. La mention en bas à droite («Chaque pièce en pages ${ }^{5}$ ») suggère qu'il pourrait s'agir d'un récapitulatif des poèmes prêts à la composition à une date donnée. Le terme «boulevard», qui accompagne Les Bienfaits de la lune et Laquelle est la vraie?, fait référence à la publication de ces deux poèmes dans Le Boulevard le 14 juin 1863. L'adjectif «inédit», entre parenthèses, qui suit les titres des neuf derniers poèmes, fournit un premier indice de datation: Les Bons Chiens a paru pour la première fois dans L'Indépendance belge le 21 juin 1865 ; le document est donc nécessairement antérieur à cette date. Un deuxième indice permet d'affiner cette datation : les cinq poèmes marqués d'une croix (La Solitude, Les Projets, La Fausse Monnaie, Le Port, Le Miroir) ont paru dans la Revue de Paris le 25 décembre 1864 . On peut penser que cette liste a été établie peu avant ou peu après cette date et qu'elle était un outil de travail destiné à faciliter le pointage des poèmes parus ou à paraître.

Dans une lettre à Armand Fraisse du 5 février 1865, Baudelaire écrit par ailleurs que son recueil de poèmes en prose serait ordonné selon une «classification particulière ${ }^{6}$ ». Le poète envisageait-il de créer des sections comme il l'a fait dans Les Fleurs du Mal? Sur l'une des listes retrouvées dans ses archives apparaissent précisément des titres de section : «Choses parisiennes», «Onéirocritie», «Symboles et moralités $»^{7}$. Le Spleen de Paris que nous ont livré Banville et Asselineau ne reflète en rien ce projet de «classification».

\section{La lettre-dédicace à Arsène Houssaye}

À la suite de Banville et Asselineau, les éditeurs du Spleen de Paris ont pris l'habitude de faire précéder le recueil de la célèbre lettre-dédicace à Arsène Houssaye, que la postérité considère comme un texte fondateur dans l'histoire du poème en prose :

\section{À Arsène Houssaye}

Mon cher ami, je vous envoie un petit ouvrage dont on ne pourrait pas dire, sans injustice, qu'il n'a ni queue ni tête, puisque tout, au contraire, y est à la fois tête et queue, alternativement et réciproquement. Considérez, je vous prie, quelles admirables commodités cette combinaison nous offre à tous, à vous, à moi et au lecteur. Nous pouvons couper où nous voulons, moi ma rêverie, vous le manuscrit, le lecteur sa lecture; car je ne suspends pas la volonté rétive de celui-ci au fil interminable d'une intrigue superflue. Enlevez une vertèbre, et les deux morceaux de cette tortueuse fantaisie se rejoindront sans peine. Hachez-la en nombreux fragments, et vous verrez que chacun peut exister à part. Dans l'espérance que quelques-uns de ces tronçons seront assez vivants pour vous plaire et vous amuser, j'ose vous dédier le serpent tout entier.

J'ai une petite confession à vous faire. C'est en feuilletant, pour la vingtième fois au moins, le fameux Gaspard de la Nuit, d'Aloysius Bertrand (un livre connu de vous, de moi et de quelques-uns de nos amis, n'a-t-il pas tous les droits à être appelé fameux?), que l'idée m'est venue de tenter quelque chose d'analogue, et d'appliquer à la description de la vie moderne, ou plutôt d'une vie moderne et plus abstraite, le procédé qu'il avait appliqué à la peinture de la vie ancienne, si étrangement pittoresque.

Quel est celui de nous qui n'a pas, dans ses jours d'ambition, rêvé le miracle d'une prose poétique, musicale sans rythme et sans rime, assez souple et assez heurtée pour s'adapter aux mouvements lyriques de l'âme, aux ondulations de la rêverie, aux soubresauts de la conscience?

C'est surtout de la fréquentation des villes énormes, c'est du croisement de leurs innombrables rapports que naît cet idéal obsédant. Vous-même, mon cher ami, n'avez-vous pas tenté de traduire en une chanson le cri strident du Vitrier, et d'exprimer dans une prose lyrique toutes les désolantes suggestions que ce cri envoie jusqu'aux mansardes, à travers les plus hautes brumes de la rue?

Mais, pour dire le vrai, je crains que ma jalousie ne m'ait pas porté bonheur. Sitôt que j'eus commencé le travail, je m'aperçus que non seulement je restais bien loin de mon mystérieux et brillant modèle, mais encore que je faisais quelque chose (si cela peut s'appeler quelque chose) de singulièrement différent, accident dont tout autre que moi s'enorgueillirait sans doute, mais qui ne peut qu'humilier

3. Un fac-similé de ce document figure dans l'édition du Spleen de Paris par Jean-Luc Steinmetz, Paris, Le Livre de poche, 2003, p. 58.

4. Jacques Dupont, art. cit., p. 49.

5. On a pu penser que cette mention n'est pas de la main de Baudelaire (voir par exemple ibid., p. 48). Nous pensons qu'elle est bien de la main du poète.

6. Charles Baudelaire, Nouvelles Lettres, Paris, Fayard, 2000, p. 96.

7. Id., CEC I, éd. cit., p. 366-367. 
profondément un esprit qui regarde comme le plus grand honneur du poète d'accomplir juste ce qu'il a projeté de faire. Votre bien affectionné,

$$
\text { C. B. } 8
$$

Est-il légitime de faire figurer la lettre-dédicace à Houssaye en tête du Spleen de Paris? Bel exemple de captatio benevolentia, ce texte a paru le 26 août 1862 dans La Presse, dont la partie littéraire était dirigée par Houssaye. Il sert d'introduction à la première série de poèmes en prose que Baudelaire a publiés dans ce quotidien en août-septembre 1862 .

Il n'est pas rare, au XIXe siècle, que l'auteur d'un feuilleton fasse précéder son œuvre d'une lettre ouverte au directeur de publication ou au rédacteur en chef, sur le ton du badinage mondain et spirituel. Ainsi, dans Le Gaulois du 23 mai 1872, le feuilleton inaugural du Salon de 1872 de Barbey d'Aurevilly s'ouvre par une lettre à Edmond Tarbé des Sablons, directeur-gérant du quotidien. Romancier et critique littéraire reconnu, d'Aurevilly n'était pas réputé pour sa critique d'art. Ironisant sur l'innocence virginale qu'il est censé avoir en la matière, le futur auteur des Diaboliques fait mine d'accepter de relever un défi qu'on lui lance :

\section{À Monsieur le directeur du Gaulois Un ignorant au Salon}

\section{I.}

C'est moi, monsieur, qui suis cet ignorant-là.

Je ne m'en vante pas, mais j' ai cru devoir vous le dire quand vous êtes venu à moi, et je crois devoir le dire tout aussi rondement au public, qui peut-être n'y viendra pas. Ni à vous, ni à lui, je ne veux vendre chat en poche. Vous, monsieur, vous n'avez pas eu peur, - le public sera-t-il aussi brave que vous? - de ma déclaration d'ignorance, quand il s'est agi de faire le Salon dans Le Gaulois ressuscité, et Gaulois vous-même, vous avez gaiement pensé : «Tiens, comme cela, ce sera plus drôle! Celui-ci ne sait rien de tout ce que les autres savent peut-être trop... Si j'essayais!»

Et pourquoi pas? En général les ingénues sont intéressantes quand elles ne sont pas trop bêtes. L'ignorance donne parfois aux impressions de la fraîcheur et de l'originalité. [...] C'est une carte à jouer!

Jouons-la donc, monsieur! Nous ne prenons pas les gens en traître. Tenez! au petit bonheur de mes impressions et de mon ignorance, et au hasard de la fourchette!

Pourvu que cette diable de fourchette ait des dents 9 .
La lettre-dédicace de Baudelaire à Houssaye relève du même genre. Elle pratique volontiers l'autodérision : le premier paragraphe évoque allusivement, sur le mode de l'ironie masochiste, la mutilation des Fleurs du Mal après le procès de 1857 («Nous pouvons couper où nous voulons »). (Euvre de circonstance, liée à la publication de trois séries de poèmes en prose dans La Presse en aoûtseptembre 1862, elle est peut-être aussi une commande que Houssaye aurait faite à Baudelaire, contraint de sacrifier aux usages de la presse de son temps. D'où le ton narquois et faussement complaisant du texte. Un billet de Baudelaire à Arsène Houssaye, daté du 18 août 1862, tend à confirmer cette hypothèse :

il m'a semblé que tout cela [les poèmes en prose à paraître] tombait sur la tête du lecteur, dès le premier feuilleton, comme des pierres de la lune et qu'il serait bien de glisser d'abord quelques phrases explicatives. Je les ai rassemblées sous la forme d'une dédicace que je vous prie d'agréer ${ }^{10}$.

Aucun élément, dans les notes de travail de Baudelaire, ne permet d'affirmer que le poète souhaitait faire figurer la lettre-dédicace à Houssaye en tête du Spleen de Paris. La lettre-dédicace n'est pas répertoriée sur le document, transcrit ci-dessus, à partir duquel Banville et Asselineau ont établi l'ordre des poèmes. En accordant à ce texte une importance que lui dénient les papiers du poète, les premiers éditeurs du Spleen de Paris ont sans doute voulu rendre un hommage complice à Houssaye, figure éminente de la vie littéraire et du Tout-Paris, directeur de L'Artiste puis de la Revue du XIXe siècle, administrateur de la Comédie-Française de 1849 à 1856 et inspecteur des musées de province du Second Empire depuis 1857.

8. Ibid., p. 275-276

9. Jules Barbey d'Aurevilly, «Un ignorant au Salon», Le Gaulois, 23 mai 1872; rééd. dans Sensations d'art (1887), Euvres complètes, dir. P. Glaudes et C. Mayaux, Paris, Les Belles Lettres, t. II, 2006, p. 813-814. 10. Lettre vendue à Drouot-Richelieu le 3 avril 2013 et éditée par Michel Brix («Du procès des Fleurs du Mal à la composition du Spleen de Paris», dans Lire «Le Spleen de Paris», sous la direction d'André Guyaux et Henri Scepi, Paris, Presses de 1'Université Paris-Sorbonne, coll. «Colloques de la Sorbonne», 2014, p. 17). 


\section{Le dédale des préoriginales}

La plupart des poèmes du Spleen de Paris ont été publiés plusieurs fois, dans différents périodiques ${ }^{11}$. D'une version à l'autre, Baudelaire a retouché son texte. Les choix éditoriaux de Banville et Asselineau se révèlent parfois incohérents. Dans certains cas, ils aboutissent à des chimères textuelles : pour Laquelle est la vraie?, publié une première fois sous ce titre dans Le Boulevard le 14 juin 1863 et une seconde fois sous le titre L'Idéal et le Réel dans la Revue nationale et étrangère le 7 septembre 1867, les premiers éditeurs du Spleen de Paris ont retenu le texte de la seconde version et le titre de la première. De même pour Le Joueur généreux, publié une première fois sous ce titre dans le Figaro du 7 février 1864, et une seconde fois dans la Revue du XIXe siècle de juin 1866, sous le titre Le Diable.

Il peut sembler a priori logique d'établir le texte d'un poème à partir de sa dernière version publiée et de donner en notes les variantes des publications antérieures. Dans le cas de L'Invitation au voyage, publiée dans Le Présent en août 1857, dans la Revue fantaisiste en novembre 1861 et dans La Presse en septembre 1862, l'application de ce principe ne pose aucune difficulté : Baudelaire a supervisé et validé les trois publications. On peut légitimement penser qu'il considérait la dernière comme la plus aboutie.

Pour d'autres poèmes, en revanche, ce principe reste inapplicable. Si l'on examine par exemple les trois versions de La Corde, publié d'abord dans le Figaro du 7 février 1864, puis dans L'Artiste le 1er novembre 1864 et enfin dans L'Événement le 12 juin 1866, on constate que la troisième version n'est en réalité qu'une réédition de la première. La dernière version à laquelle Baudelaire a mis la main est la version publiée dans L'Artiste en 1864.

Autre exemple : Les Bons Chiens, dont il existe un manuscrit reproduit dans le catalogue de la collection Armand Godoy, et quatre préoriginales, dans L'Indépendance belge du 21 juin 1865, La Petite Revue du 27 octobre 1866, Le Grand Journal du 4 novembre 1866 et la Revue nationale et étrangère du 31 août 1867. Hospitalisé à la clinique du docteur Duval depuis juillet 1866, Baudelaire n'était plus en possession de ses facultés pour corriger les épreuves et donner le bon à tirer des trois dernières publications. Avait-il même connaissance de leur existence? La version de L'Indépendance belge a quant à elle été publiée contre la volonté du poète, comme en témoigne une lettre qu'il adresse à Narcisse Ancelle le 28 juin 1865 : «Je vous ai envoyé une bagatelle qui a été publiée malgré moi dans $L^{\prime}$ Indépendance ${ }^{12}$.» Le texte le plus fiable semble donc être celui du manuscrit.

La Belle Dorothée constitue un casse-tête philologique. Il en existe deux versions. La première, sur une épreuve corrigée datant de l'automne de 1862 . Le poème devait paraître initialement dans La Presse, mais ce projet n'a pu aboutir en raison d'une rupture de contrat entre Baudelaire et Houssaye. Une deuxième version a paru un peu plus tard, dans la Revue nationale et étrangère, le 10 juin 1863. Or elle a été remaniée à l'insu du poète, après que celui-ci eut donné le bon à tirer. Dans la version de 1862, Baudelaire écrit à l'avant-dernier paragraphe :

Dorothée est admirée et choyée de tous, et elle serait parfaitement heureuse si elle n'était obligée d'entasser piastre sur piastre pour racheter sa petite sœur qui est si belle et déjà presque mûre ${ }^{13}$.

Dans la Revue nationale et étrangère du 10 juin 1863, la même phrase est tronquée et transformée :

Dorothée est admirée et choyée de tous, et elle serait parfaitement heureuse si elle n'était obligée d'entasser piastre sur piastre pour racheter sa petite sœur qui est déjà si belle14 !

Dix jours après la sortie de presse de la revue, le 20 juin 1863, Baudelaire se plaint auprès du directeur de la revue, Gervais Charpentier, d'avoir été censuré :

Je viens de lire les deux extraits (Les Tentations et Dorothée) insérés dans la Revue nationale. J'y trouve d'extraordinaires changements introduits après mon bon à tirer. [...]

Je vous avais dit: supprimez tout un morceau, si une virgule vous déplaît dans le morceau, mais ne supprimez pas la virgule; elle a sa raison d'être. [...]

Et croyez-vous qu'il soit immoral de dire qu'une fille est mûre à onze ans, quand on sait qu' Aïscha (qui n'était pas une négresse, née sous le Tropique) était plus jeune encore alors que Mahomet l'épousa? [...]

11. Voir la liste des préoriginales dans l'édition du Spleen de Paris que nous avons procurée, Andrea Schellino et moi, dans la collection GF-Flammarion, 2017, p. 293-300.

12. Charles Baudelaire, Correspondance, éd. cit., t. II, p. 509

13. Id., Le Spleen de Paris, éd. Aurélia Cervoni et Andrea Schellino, p. 114. 14. Voir ibid., variante a. 
Si encore j'avais été prévenu à temps, j'aurais pu supprimer tout le morceau 15 .

L'histoire des mutilations et des rapiéçages de La Belle Dorothée rebondit en 1869, à l'occasion de la première édition du Spleen de Paris. Banville et Asselineau, qui avaient eu connaissance de la polémique entre Baudelaire et Charpentier, reprennent le texte de la Revue nationale et étrangère de juin 1863 en modifiant la dernière partie de la phrase selon ce qu'ils croient être le vœu de l'auteur :

Dorothée est admirée et choyée de tous, et elle serait parfaitement heureuse si elle n'était obligée d'entasser piastre sur piastre pour racheter sa petite sœur qui a bien onze ans, et qui est déjà mûre, et si belle16!

Eu égard à cette histoire éditoriale compliquée, il semble prudent d'adopter le texte d'octobre 1862, resté à l'état d'épreuve corrigée.

Dans la future édition du Spleen de Paris dans la Bibliothèque de la Pléiade, on procédera pour chaque poème à une enquête dans les papiers et la correspondance de Baudelaire afin de déterminer quel est le texte le plus respectueux des volontés du poète - le moins frelaté.

\section{L'établissement du texte}

Banville et Asselineau ont pris quelques libertés avec le texte de Baudelaire, considérant qu'il comportait des coquilles et des imperfections typographiques, voire stylistiques. Fruits d'une lecture interprétative, certaines de leurs interventions portent atteinte au sens de la phrase ou à sa musicalité. On se bornera ici à analyser trois exemples suggestifs.

Dans Les Veuves, dont il n'existe qu'une préoriginale, publiée dans La Presse le 27 août 1862, Baudelaire écrit :

Il m'est arrivé une fois de suivre pendant de longues heures une vieille affligée de cette espèce [...]. Je sais dans quel misérable café et de quelle façon elle déjeuna. Je la suivis au cabinet de lecture; et je l'épiai longtemps pendant qu'elle cherchait dans les gazettes $[\ldots]^{17}$.

Dans l'édition du Spleen de Paris de 1869, Banville et Asselineau ont introduit une négation, un ne explétif précisément, qui donne à la deuxième phrase un tour faussement élégant et en brouille le sens :
Il m'est arrivé une fois de suivre pendant de longues heures une vieille affligée de cette espèce [...]. Je ne sais dans quel misérable café et de quelle façon elle déjeuna. Je la suivis au cabinet de lecture; et je l'épiai longtemps pendant qu'elle cherchait dans les gazettes $[\ldots]^{18}$.

Le sens de la préoriginale est pourtant clair : par la formule affirmative «Je sais », le poète urbain, en quête de nouvelles expériences esthétiques, revendique la liberté de prendre en filature une inconnue. Le texte de la préoriginale a été rétabli pour la première fois par Jean-Luc Steinmetz dans son édition du Spleen de Paris (2003) ${ }^{19}$.

L'Invitation au voyage en prose offre un exemple de correction typographique injustifiée. Dans la dernière version du poème publiée du vivant de Baudelaire, le 24 septembre 1862 dans La Presse, on relève une virgule entre «revenez-y» et «de Sumatra» :

et de toutes choses, de tous les coins, des fissures des tiroirs et des plis des étoffes s'échappe un parfum singulier, un revenez-y, de Sumatra, qui est comme l'âme de l'appartement $^{20}$.

Cette virgule n'apparaît pas dans l'édition de 1869 : «un revenez-y de Sumatra »21. Pourquoi cette suppression, entérinée par la tradition? Si la virgule ne change rien au sens, elle a son importance du point de vue du rythme : elle introduit une pause. Placé entre virgules, le complément de nom, «de Sumatra», résonne comme une note précieuse.

La première phrase des Fenêtres, enfin, fait l'objet d'un débat entre les éditeurs du Spleen de Paris depuis 1869. L'unique préoriginale, publiée dans la Revue nationale et étrangère le 10 décembre 1863, donne le texte suivant :

Celui qui regarde au dehors à travers une fenêtre ouverte, ne voit jamais autant de choses que celui qui regarde une

15. Charles Baudelaire, Correspondance, éd. cit., t. II, p. 307.

16. Id., EC I, éd. cit., p. 317.

17. Id., Le Spleen de Paris, éd. Aurélia Cervoni et Andrea Schellino, p. 69-70. Je souligne.

18. Id., EEC I, éd. cit., p. 293. Je souligne.

19. Id., Le Spleen de Paris (Petits poèmes en prose), édition présentée, établie et annotée par Jean-Luc Steinmetz, Paris, Librairie générale française, coll. «Le Livre de poche», 2003, p. 47.

20. Id., Le Spleen de Paris, éd. Aurélia Cervoni et Andrea Schellino, p. 88. 21. Id., $E C$ I, éd. cit., p. 302. 
fenêtre fermée. Il n'est pas d'objet plus profond, plus mystérieux, plus fécond, plus ténébreux, plus éblouissant qu'une fenêtre éclairée d'une chandelle. Ce qu'on peut voir au soleil est toujours moins intéressant que ce qui se passe derrière une vitre. Dans ce trou noir ou lumineux vit la vie, rêve la vie, souffre la vie 22 .

À la première ligne, jugeant que «au» était une coquille pour «du», Banville et Asselineau ont corrigé « au dehors » en «du dehors » : «Celui qui regarde $d u$ dehors à travers une fenêtre ouverte 23 ...» Cette correction soulève la question du point de vue : le narrateur est-il lui-même à l'intérieur ou l'extérieur 24 ? Regarde-t-il la «fenêtre éclairée d'une chandelle» depuis une mansarde située au dernier étage ou depuis la rue, situation typique dans les romans du XIXe siècle? Le contexte semble plaider en faveur de la première hypothèse. Dans la suite du poème, Baudelaire écrit : «Par delà des vagues de toits, j'aperçois une femme mûre, ridée déjà, pauvre, toujours penchée sur quelque chose, et qui ne sort jamais ${ }^{25}$. » Le complément circonstanciel «Par delà des vagues de toits » suggère que le poète se situe en altitude, qu'il regarde donc «au dehors » depuis sa chambre. Mais le sens du texte garde une part d'indécidable. Et en l'absence de manuscrit, il est difficile de trancher.
S'il est possible de résoudre la plupart des questions d'établissement du texte en portant une attention particulière aux préoriginales, la question de l'architecture du Spleen de Paris reste pendante. L'ordre des poèmes tel que nous le connaissons ne rend pas compte du caractère inachevé du recueil. Une édition numérique, qui permettrait entre autres de confronter simultanément les différentes versions d'un même poème et de visualiser la chronologie des publications préoriginales, constitue à cet égard l'un des projets les plus prometteurs.

22. Charles Baudelaire, EC I, éd. cit., p. 339 et 1342 (Claude Pichois indique qu'il corrige le texte de l'édition du Spleen de Paris de 1869 en se fondant sur la préoriginale). Voir aussi Le Spleen de Paris, éd. Aurélia Cervoni et Andrea Schellino, p. 154. Je souligne.

23. Euvres complètes de Charles Baudelaire, Paris, Michel Lévy, t. Iv, 1869, p. 109. Je souligne.

24. Andrea Del Lungo a fait le point sur ce débat ( $\ll$ Sens du regard et construction identitaire. Sur Les Fenêtres», L'Année Baudelaire, no 17, 2013, p. 115-128).

25. Charles Baudelaire, CEC I, éd. cit., p. 339.

Docteur en littérature française, AURÉLIA CERvoni est l'auteur de deux ouvrages : Théophile Gautier devant la critique 1830-1872 (Classiques Garnier, 2016) et Pétrus Borel (Sorbonne Université Presses, 2020). Elle a collaboré à l'édition des Euvres complètes de Rimbaud de la Bibliothèque de la Pléiade (2009). Secrétaire de rédaction de L'Année Baudelaire, elle a édité Le Spleen de Paris (2017) et Les Paradis artificiels (2020) pour la collection GF-Flammarion. 